\title{
TEXTURES IN PURE SHEAR DEFORMED ROCK SALT
}

\author{
W. SKROTZKI, ${ }^{1}$ K. HELMING ${ }^{2}$ H.-G. BROKMEIER, ${ }^{3}$ \\ H.-J. DORNBUSCH ${ }^{4}$ and P. WELCH ${ }^{5 *}$ \\ ${ }^{1}$ Institut für Kristallographie und Festkörperphysik der TU Dresden, \\ 01062 Dresden, Germany \\ ${ }^{2}$ Institut für Metallkunde und Metallphysik der TU Clausthal, Großer Bruch 23, \\ 38678 Clausthal-Zellerfeld, Germany \\ ${ }^{3}$ Institut für Metallkunde und Metallphysik der TU Clausthal, Außenstelle am \\ GKSS-Forschungszentrum Geesthacht GmbH, Max-Planck-Straße, \\ 21502 Geesthacht, Germany \\ ${ }^{4}$ Institut für Geologie und Dynamik der Lithosphäre der Universität Göttingen, \\ Goldschmidtstr. 3, 37077 Göttingen, Germany and \\ ${ }^{5}$ Institut für Metallkunde und Metallphysik der TU Clausthal, Großer Bruch 23, \\ 38678 Claustal-Zellerfeld, Germany \\ (Received 30 October 1994; in final form 20 December 1994)
}

Texture formation in pure shear deformed rock salt has been studied by neutron diffraction. The textures developed are comparable to those of rolled face-centered cubic metals with high stacking fault energy. At room temperature the texture consists of a strong $S$ and copper and a weaker brass component. It is replaced by static and/or dynamic recrystallization by a strong cube and a subordinate Goss component. Comparison of the experimental textures with simulations based on different models shows that the low temperature high strain deformation texture can be qualitatively well explained by the Taylor model using slip on $\{110\}\langle 110\rangle,\{100\}\langle 110\rangle$ and $\{111\}\langle 110\rangle$ systems with equal critical resolved shear stresses. Relaxation with increasing temperature introduces the cube and Goss component, which may represent the nuclei for recrystallization. The study shows that texture simulations on salt without considering recrystallization lead to misleading conclusions.

KEY WORDS: Rock salt, pure shear deformation, deformation texture, recrystallization texture, texture modelling, plastic anisotropy.

\section{INTRODUCTION}

Salt deforms by slip on $\{110\}$ and $\{100\}$ planes in $\langle 110\rangle$ direction. $\{111\}$ may be activated, too, in particular as cross-slip plane (Matucha 1968, Strunk 1975, Bhagavanraju and Strunk 1979). The critical resolved shear stresses (CRSS) for the activation of these slip systems are different. This plastic anisotropy depends on divalent doping, temperature, strain rate and ionicity of the material. For salt at low temperatures $\{110\}$ is the primary slip plane (Skrotzki and Haasen 1981).

There exist six crystallographically equivalent $\{110\}\langle 110\rangle$ and $\{100\}\langle 110\rangle$ slip systems in each case, however, only two and three are independent, respectively. Thus, in order

*present address: Ostpreußenstr. 14, 61381 Friedrichsdorf 
to deform a polycrystalline aggregate homogeneously, according to the von Mises criterion (von Mises 1928) requiring five independent slip systems both types of families have to be activated simultaneously. This should lead to difficulties at low temperatures where the plastic anisotropy increases strongly due to the high Peierls potential for secondary slip (Skrotzki and Suzuki 1983). Nevertheless, the temperature dependence of the yield stress of polycrystals indicates that the activation of secondary slip systems is necessary (Figure 1, Skrotzki et al. 1981, Skrotzki and Haasen 1984). However, their number and contribution to strain is not clear.

With increasing temperature the plastic anisotropy decreases and above a certain temperature it is practically unity. In addition cross slip and climb as well as dynamic recrystallization become operative. To account for these processes as well as the plastic anisotropy different models (relaxed constraints (van Houtte 1988) and selfconsistent (Molinari et al. 1987, Molinari and Toth 1994)) have been developed which yield deviations from the full constraints Taylor model (Taylor 1938) requiring strain homogeneity towards the Sachs model (Sachs 1928) based on stress equilibrium. This has been demonstrated recently by Skrotzki (1994).

It is the aim of this paper to extend texture research on salt to pure shear deformation, a deformation mode which is more suitable to check models on texture formation because of its orthorhombic symmetry. The experimental results will be compared with different models on texture formation.

Salt may be considered as model material for intermetallics, ceramics and rockforming minerals which in general exhibit a high plastic anisotropy, too. Experimental deformation of such materials under normal conditions is difficult, if not impossible, and therefore conclusions derived from model materials are very helpful.

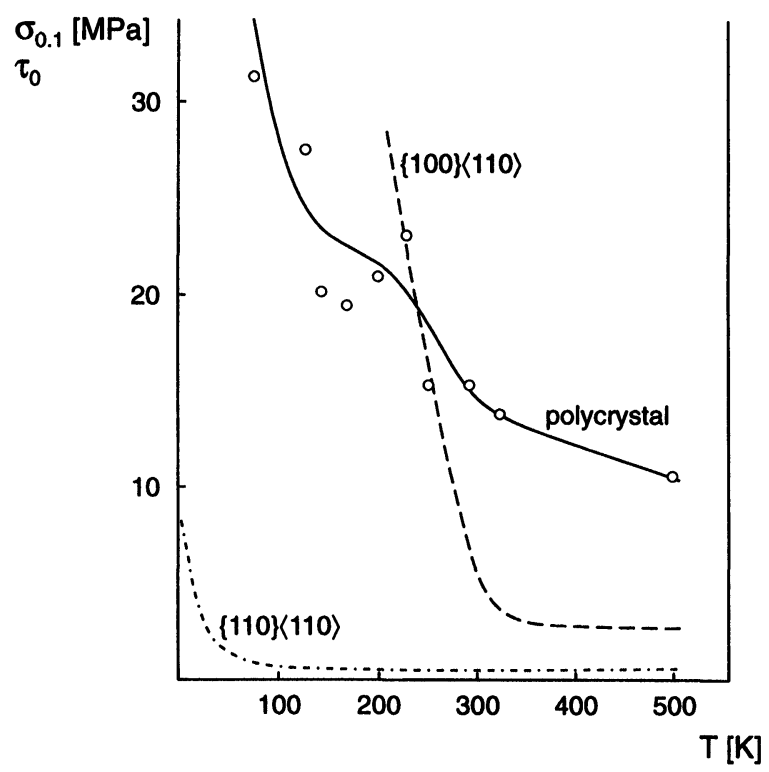

Figure 1 Temperature dependence of the critical flow stress at $0.1 \%$ strain (solid line) and the CRSS for $\{110\}\langle 110\rangle$ and $\{100\}\langle 110\rangle$ slip (dashed lines) in poly- and monocrystalline $\mathrm{NaCl}$, respectively. $\left(\dot{\varepsilon}=5 \cdot 10^{-5} \mathrm{sec}^{-1}\right.$, from Skrotzki et al. 1981). 


\section{EXPERIMENTAL DETAILS}

Deformation experiments have been carried out on coarse-grained natural salt from the Asse Mine, Northern Germany, with a grain size in the order of $\mathrm{cm}$. Pure shear deformation was approximated by extrusion of cylindrical samples (diameter $7.5 \mathrm{~cm}$, length $10 \mathrm{~cm})$ through a rectangular die $(0.7 \mathrm{~cm} \times 3.5 \mathrm{~cm})$. Lubrication at temperatures below $300^{\circ} \mathrm{C}$ was accomplished by a thin lead cover. The strain rate was in the order of $1 \mathrm{sec}^{-1}$ (Skrotzki and Welch 1983). There has been some adiabatic heating during this high strain rate deformation.

Complete pole figures for $\{200\},\{220\}$ and $\{111\}$ reflections have been measured by neutron diffraction at the Hahn-Meitner-Institute in Berlin and at the GKSS-Research Centre in Geesthacht, Germany. Orientation distribution functions (ODFs) have been calculated according to the iterative series expansion method given by Dahms and Bunge (1989) and Dahms (1992). Texture components have been quantitatively determined with the component fit developed by Helming and Eschner (1990).

The textures have been simulated by using 36 orientations randomly distributed in the Euler space. This number is sufficient because only end positions are looked for. This has been checked for certain cases with 400 orientations. The total strain achieved after 100 steps of $5 \%$ is $99.4 \%$. The slip systems used are either $\{110\}\langle 110\rangle$ and $\{100\}\langle 110\rangle$ or additionally $\{111\}\langle 110\rangle$. Their relative CRSS chosen are: (A1) $1 / 10$, (A2) $1 / 1$ and (A3) $1 / 1 / 1$.

In the full constraints (FC) Taylor model those 5 independent slip systems are activated, which require the least amount of work. Relaxed constraints (RC) are introduced by adding pseudo slip systems in the sample coordinate system causing certain components of the fixed macroscopic strain tensor to be released in the crystal. Deformation for these components is not homogeneous any longer. Incompatibilities necessarily arising at the grain boundaries are justified by the fact that in the case of special grain shapes only small areas of the specimen are affected. The grain shape used here is a long grain, the shear relaxed is $\varepsilon_{13}$.

\section{RESULTS}

\section{Experimental Textures}

Extrusion of salt through an extreme rectangular die leads to a texture comparable to that generally observed in rolled face-centered cubic (FCC) metals with high stacking fault energy (SFE). In the ODF representation this texture after low temperature deformation is characterized by the $\alpha$ - and $\beta$-fibre which commonly has a maximum at the $\{110\}\langle 112\rangle$ (brass $\mathrm{Bs}$ ) and maxima at the $\{112\}\langle 111\rangle$ (copper $\mathrm{Cu}$ ) and $\{123\}\langle 634\rangle(\mathrm{S})$ component, respectively (Figure 2c, Bacroix and Jonas 1988). The room temperature "deformation texture" of salt is dominated by these components (Figure 2a, Table 1).

Dynamic recrystallization at higher temperatures leads to the $\{100\}\langle 100\rangle$ (cube C) and subordinate $\{110\}\langle 100\rangle$ (Goss G) component (Figure 2b). This "recrystallization texture" also develops statically by holding room temperature deformed salt at room temperature. Figure $3 a$ shows the textural change after a period of about 10 years. In contrast, room temperature storage of dynamically recrystallized salt does not lead to a drastic change (Figure 3b). 

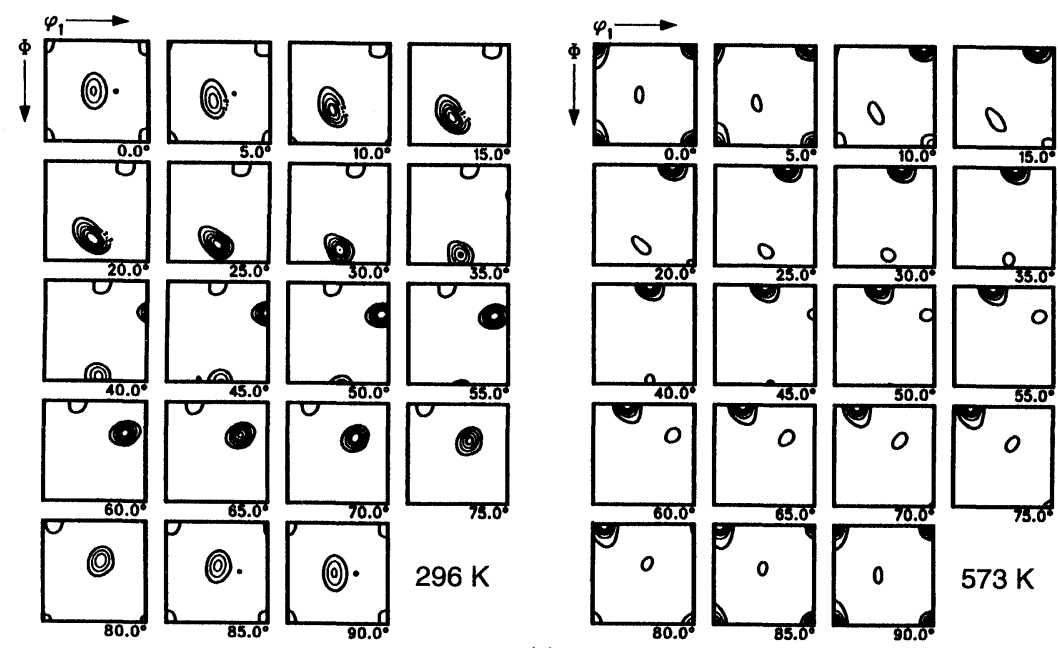

(a)

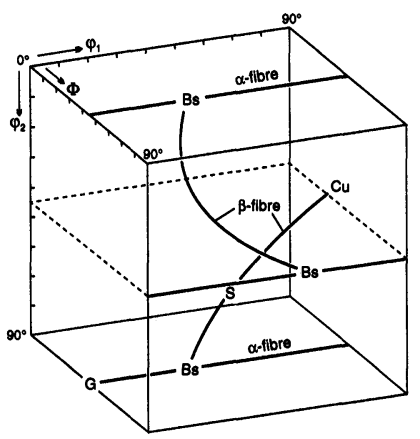

(b)
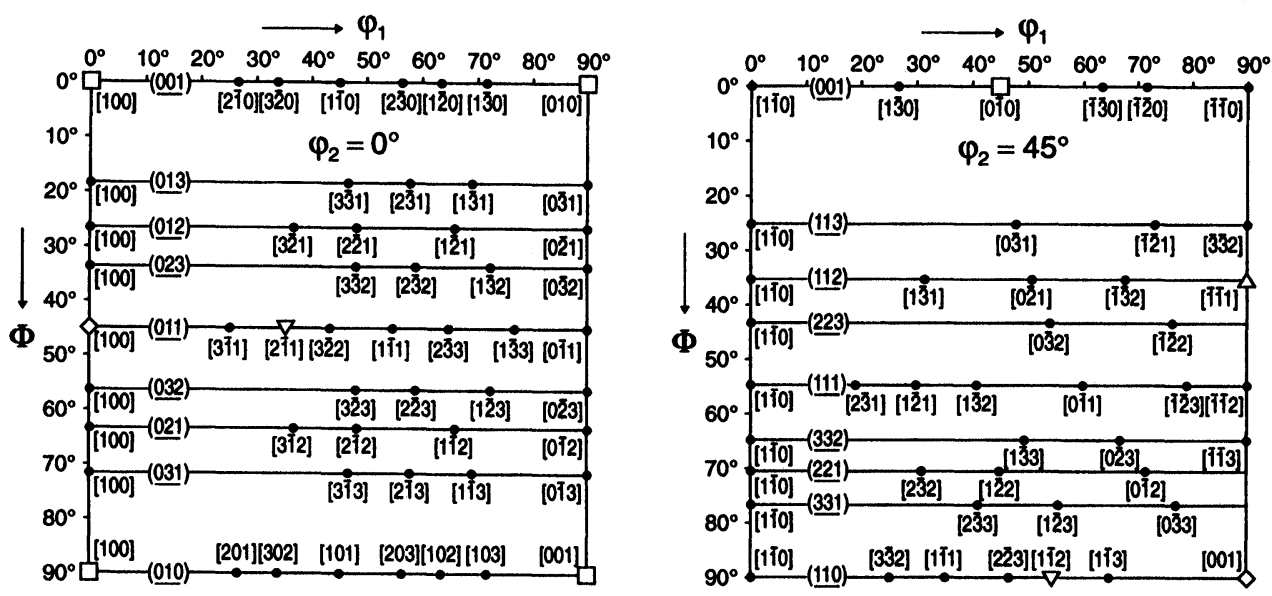

(c)

Figure 2 (a) Complete ODF contour plots in $\varphi_{2}$ sections of salt deformed in pure shear at $296 \mathrm{~K}$ and $573 \mathrm{~K}(296 \mathrm{~K}: 2.5,7.5, \ldots$ m.r.d., $\max =26.84, \min =-1.27 ; 573 \mathrm{~K}: 5,15, \ldots$. m.r.d., $\max =55.32, \min =-2.14$; degree of series expansion $\mathrm{L}_{\max }=23$ ). (b) Position of the $\alpha$ - and $\beta$-fibre in the Euler space. (c) Ideal orientation charts for cubic symmetry: $\Delta$ Copper $(\mathrm{Cu}), \nabla$ Brass (Bs), \& Cube (C), $\diamond$ Goss (G) component. 
Table 1 Main components of the experimental (room temperature) and simulated deformation textures. The Euler angles of the ideal components are given in brackets.

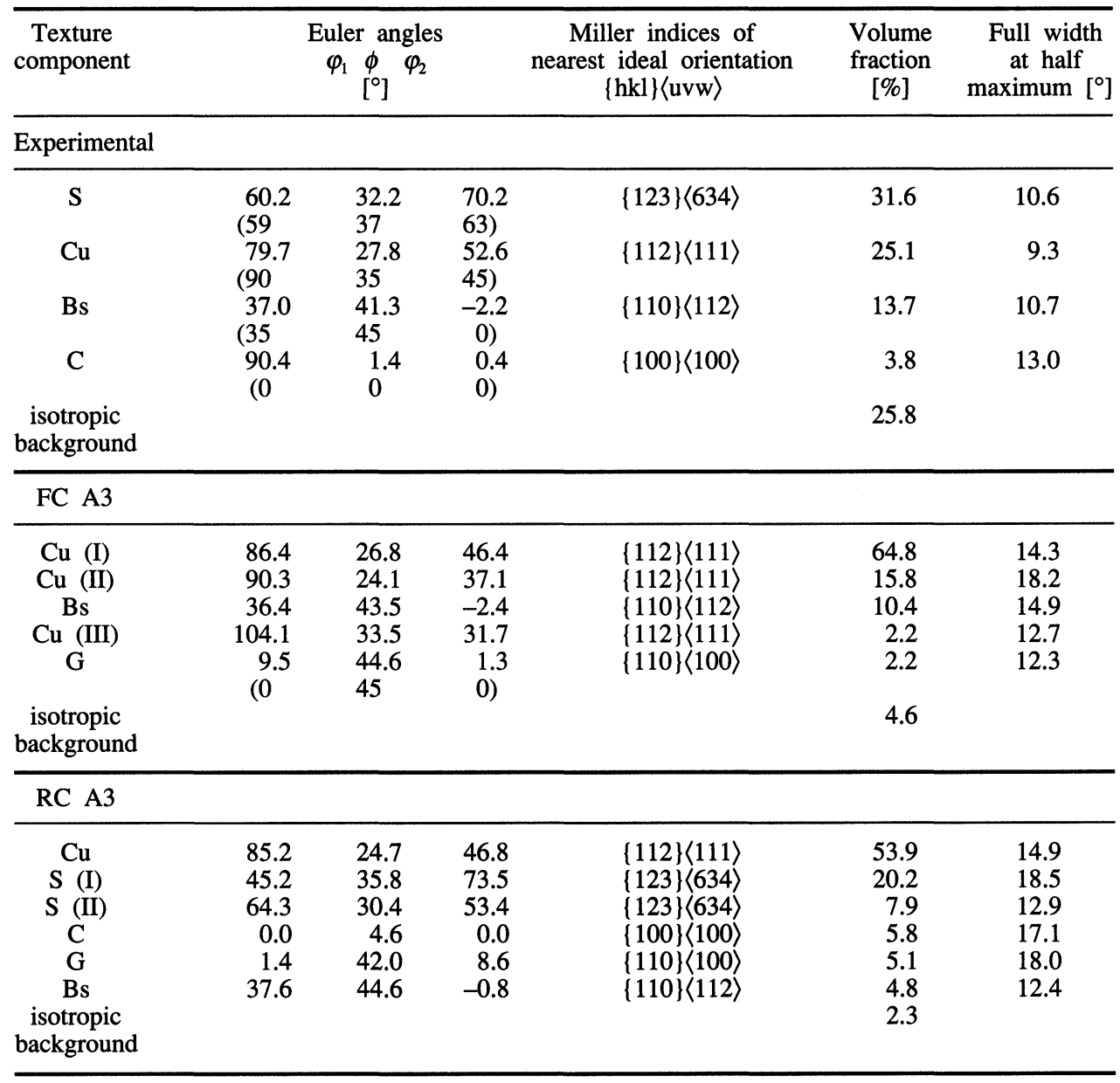

The same holds for the change of microstructure. Figures $4 \mathrm{a}-\mathrm{c}$ show the microstructure at different times after room temperature deformation. Just after deformation primary recrystallization starts extinguishing the strongly elongated deformation structure. Recrystallization preferentially begins at the grain boundaries. The new grains often have a cubic shape with a cube plane surface and therefore clearly show the orientation of the cube component. The isomorphic growth ceases during grain coarsening. Primary and secondary recrystallization leads to a very inhomogeneous microstructure.

At higher temperatures dynamic recrystallization leads to a coarser grained microstructure (Figure 4d), which only slightly coarsens by storing at room temperature. Due to differences in stress the grain size depends on the grain orientation. The microstructure gets equiaxed when the impurity-loaded host grain boundaries become mobile (Skrotzki and Welch 1983). 

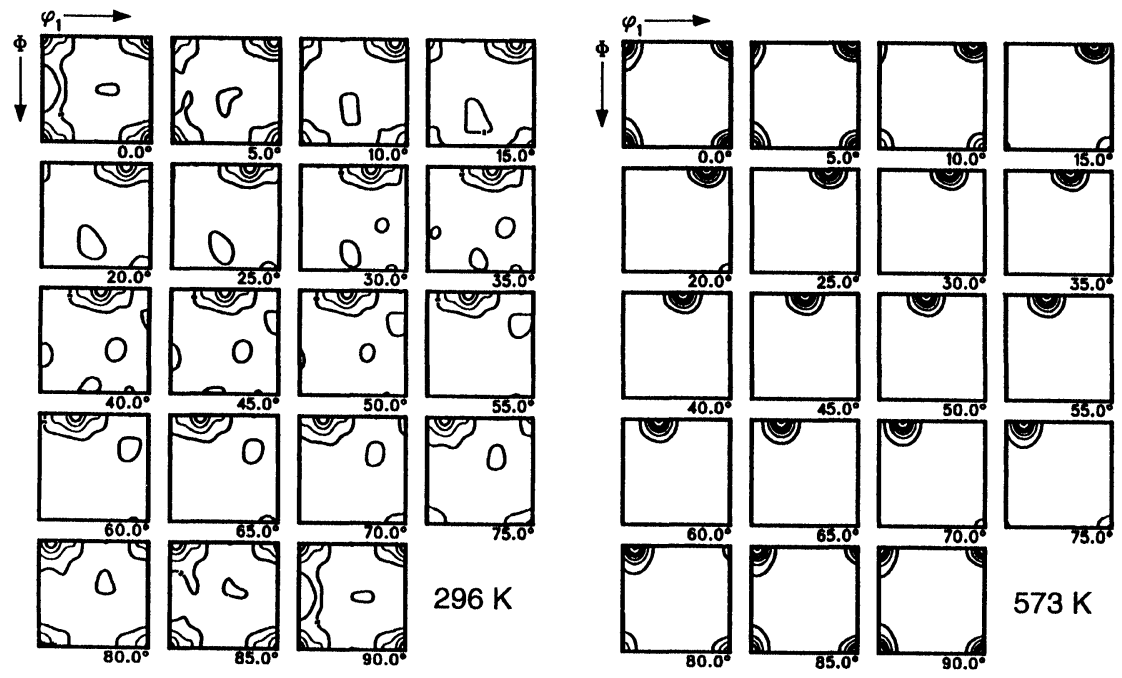

Figure 3 Complete ODF plots of the same samples as in Figure 2 measured after ten years storage at room temperature $(296 \mathrm{~K}: 2,6, \ldots$. m.r.d., $\max =17.13, \min =-1.26 ; 573 \mathrm{~K}: 5,15, \ldots$. m.r.d., $\max =57.94, \min =-0.53$, degree of series expansion $\mathrm{L}_{\max }=23$ ).

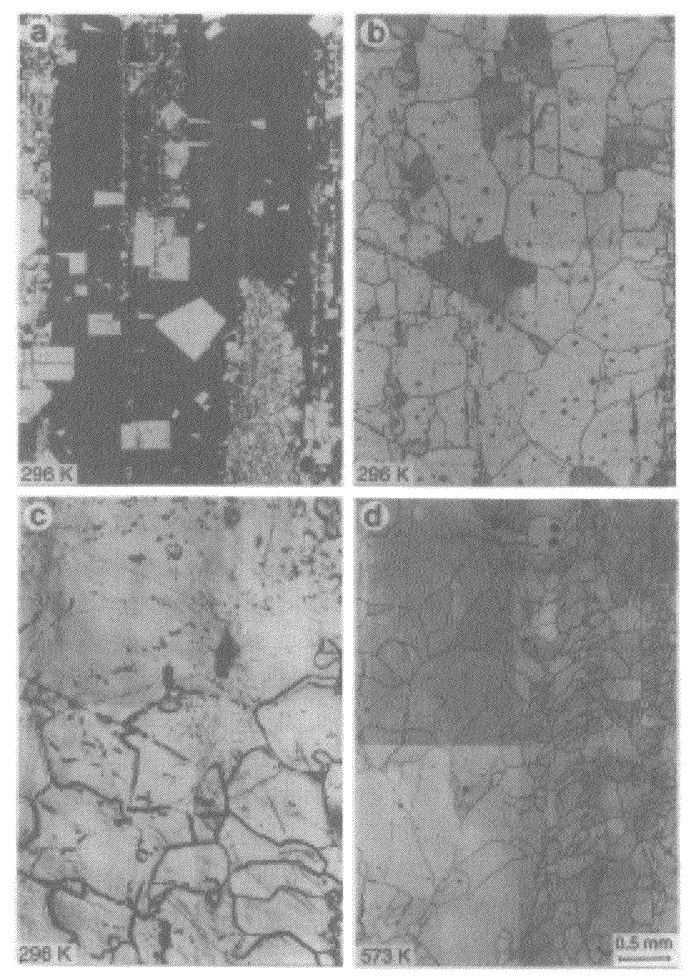

Figure 4 (a) Microstructure of a room temperature deformed sample just after deformation (dark areas are non-recrystallized deformed grains), (b) Fully recrystallized microstructure $1 / 2$ year and (c) 10 years later. (d) Dynamically recrystallized microstructure just after deformation at $300^{\circ} \mathrm{C}$. (View on flat side with extrusion direction vertical). 


\section{Model Textures}

The deformation textures have been simulated using the FC and RC (long grains) Taylor models developed by van Houtte (1988). The input parameters used are the number of different slip systems and the plastic anisotropy.

The simulations (Figure 5, Table 1) show that models using only $\{110\}$ and $\{100\}$ slip do not explain the deformation texture observed. The $\mathrm{Cu}, \mathrm{S}$ and $\mathrm{Bs}$ component can only be introduced by adding $\{111\}$ slip. Models assuming plastic isotropy (A3)

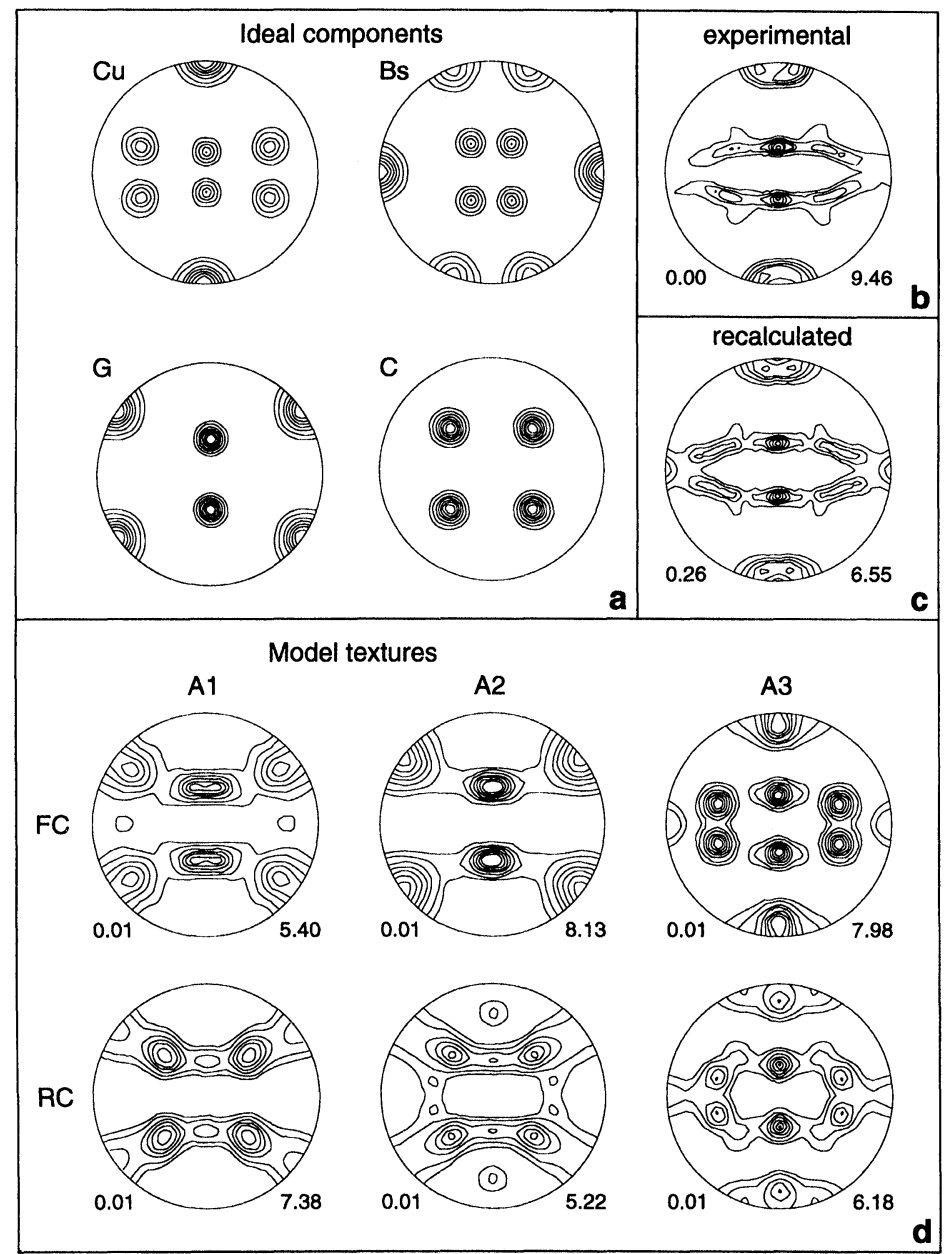

Figure $5\{111\}$ pole figures of experimental (room temperature) textures (b) and those simulated (d) according to the full constraints (FC) and relaxed constraints (RC) model for long grains using different plastic anisotropies: $A 1=\operatorname{CRSS}\{110\} / \operatorname{CRSS}\{100\}=1 / 10 ;$ A $2=$ CRSS $\{110\} / \operatorname{CRSS}\{100\}$ $=1 / 1 ; \mathrm{A} 3=\operatorname{CRSS}\{110\} / \operatorname{CRSS}\{100\} / \operatorname{CRSS}\{111\}=1 / 1 / 1$. (contours: $0.5,1,2,3,5,6,8$ m.r.d.; min: left, max: right bottom; extrusion direction = rolling direction: vertical, transverse direction: horizontal, normal direction: perpendicular to sheet). (c) Experimental texture recalculated with the main components given in Table I. (a) Position of ideal components: Copper (Cu), Brass (Bs), Goss (G), Cube (C) $(1,2.5,5,7.5,10,12.5,15,20$ m.r.d.; $\min =0, \max =20.2$, volume fraction $=100 \%$, full width at half maximum $=15^{\circ}$ ). 
describe best the deformation texture. Relaxing the conditions (FC $\rightarrow$ RC) by increasing the deformation temperature leads to an increase in $G$ and the appearance of C. These deformation components seem to be the nuclei for fast recrystallization, which could not be totally suppressed even at room temperature (Figure 4a, Table 1). There may be considerable deviations from the ideal components. However, experimental and model textures tend into the same direction. Modelling towards a better agreement with experiment is in progress.

\section{DISCUSSION}

The present investigations show that the deformation and recrystallization textures of salt are similar to those of FCC metals with high SFE, like aluminum. This has already been shown earlier for extension and compression (Skrotzki 1994). In this case the deformation texture could be explained by the FC Taylor model using slip on the commonly observed $\{110\}\langle 110\rangle$ and $\{100\}\langle 110\rangle$ systems. The plastic anisotropy did not play a part. In contrast, the pure shear experiments show that this explanation does not hold any more. To account for the $\mathrm{Cu}, \mathrm{S}$ and $\mathrm{Bs}$ component found in this deformation mode $\{111\}\langle 110\rangle$ slip has to be considered, too. This is not surprising as the $\{111\}$ plane has been frequently observed as cross slip plane (Matucha 1968, Strunk 1975). In the extrusion experiments performed the stresses and strains are high enough to initiate cross slip. Cross slip may be also favoured by the stress state in a polycrystalline aggregate. Thus, the plastic anisotropy at the onset of deformation vanishes with increasing strain. A similar effect seems to happen in FCC metals between the primary $\{111\}\langle 110\rangle$ and the secondary $\{110\}\langle 110\rangle,\{100\}\langle 110\rangle$ and $\{112\}\langle 110\rangle$ slip systems. Taking into account the non-octahedral systems the Bs and $\mathrm{C}$ component in hot plane strain compressed non-recrystallized $\mathrm{Al}$ alloys may be explained (Maurice and Driver 1994). Similar to salt the C deformation component is assumed to contribute the nuclei for recrystallization.

Recrystallization in salt occurs dynamically and statically, even at room temperature. It obliterates the temperature dependence of the deformation texture. Recrystallization is strongly affected by impurities. While divalent impurities impede grain boundary migration (Guillope and Poirier 1979) fluid phases seem to have the opposite effect (Urai et al. 1986). Fluid phases which probably are mainly present as a thin grain boundary layer lead to a higher diffusivity and may therefore be responsible for the fast isomorphic static grain growth observed at low temperatures. Detailed investigations are under way to study the kinetics of the static recrystallization.

The texture development in salt also compares well with that observed in rolled $\mathrm{AgCl}$ (Richman and Liu 1965). In this material the strong $\mathrm{Cu}$ deformation component at low temperatures is replaced by Bs at high temperatures. Annealing low temperature deformed $\mathrm{AgCl}$ at high temperatures leads to the $\mathrm{C}$ recrystallization texture.

The comparison of NaCl-type ionic crystals of different ionicity, i.e. different plastic anisotropy (Skrotzki and Haasen 1981), with high SFE FCC metals clearly shows that texture development during high strain deformation does not depend on the plastic anisotropy existing at the onset of deformation of single crystals. High strain deformation is dominated by cross slip, i.e. the main slip parameter is the Burgers vector and this is the same for FCC metals and NaCl-type ionic crystals. However, there is a big difference to FCC metals with low SFE. In these metals mechanical twinning contributes to texture formation. Thus, low temperature deformed 
ionic crystals should only be compared with high temperature deformed low SFE metals, provided recrystallization is suppressed (Bacroix and Jonas 1988).

It should be emphasized that special care has to be taken when comparing simulated deformation textures with those of easily recrystallizing materials. If recrystallization is not taken into account such a comparison will lead to misleading conclusions as is the case in the paper by Wenk et al. (1988). There the recrystallization component is fully assigned to deformation supporting the self-consistent approach. However, this theory similar to the RC Taylor model seems only to be capable of explaining deformation textures at temperatures where climb-controlled recovery processes become operative.

\section{Acknowledgements}

Thanks are due to Prof. P. van Houtte for providing his texture simulation program and giving helpful advices. Help in ODF calculations by Dr. M. Dahms and data search by Dr. H. Klein is gratefully acknowledged. This work has been supported by the Deutsche Forschungsgemeinschaft through grants Sk 21/7 (H.-J. D.) and He 2104/1 (K.H.) and the Bundesministerium für Forschung und Technologie through 03BU3CLA F.4-K19 (H.-G. B.).

\section{References}

Bacroix, B. and Jonas, J. J. (1988). In: Proc. $8^{\text {th }}$ Int. Conf. on Textures of Materials, p. 403-413, Eds. J. S. Kallend and G. Gottstein, The Metallurgical Society.

Bhagavanraju, I. V. K. and Strunk (1979). H. Phys. Stat. Sol. (a) 53, 211-221.

Dahms, M. (1992). J. Appl. Cryst. 25, 258-267 (1992).

Dahms, M. and Bunge, H.-J. (1989). J. Appl. Cryst. 22, 439-447.

Guillope, M. and Poirier, J. P. (1979). J. Geophys. Res. 84, 5557-5567.

Helming, K. and Eschner, Th. (1990). Cryst. Res. Technol. 25, K 203-208.

Matucha, K.-H. (1968). Phys. Stat. Sol. 26, 291-310.

Molinari, A., Canova, G. R. and Ahzi, S. (1987). Acta metall. 35, 2983-2994.

Molinari, A. and Toth, L. S. (1994). Acta metall. mater. 42, 2453-2458.

Maurice, Cl. and Driver, J. H. (1994). Mater. Sci. Forum 157-162, 807-812.

Richman, R. H. and Liu, Y. C. (1965). Trans. Met. Soc. AIME 233, 1269-1275.

Sachs, G. (1928). Z. Ver. Dtsch. Ing. 72, 734-736.

Skrotzki, W. and Haasen, P. (1981). J. Physique 42, C3-119-148.

Skrotzki, W., Frommeyer, G. and Haasen, P. (1981). Phys. Stat. Sol. (a) 66, 219-228.

Skrotzki, W. and Suzuki, T. (1983). Rad. Eff. 74, 315-322.

Skrotzki, W. and Welch, P. (1983). Tectonophysics 99, 47-61.

Skrotzki, W. and Haasen, P. (1984). In: Deformation of Ceramics II, p. 429-444, Eds. R. E. Tressler and R. C. Bradt, Plenum Publishing Corporation.

Skrotzki, W. (1994). In: Textures of Geological Materials, p. 167-186, Eds. H. J. Bunge, S. Siegesmund, W. Skrotzki and K. Weber, DGM Informationsgellschaft Verlag, Oberursel.

Strunk, H. (1975). Phys. Stat. Sol. (a) 28, 119-126.

Taylor, G. I. (1938). J. Inst. Metals 62, 307-324.

Urai, J. L., Spiers, C. J., Zwart, H. J. and Lister, G. S. (1986). Nature 324, 554-557.

van Houtte, P. (1988). Textures and Microstructures 8, 313-350.

von Mises, R. (1928). Z. Angew. Math. Mech. 8, 161-185.

Wenk, H.-R., Canova, G. R., Molinari, A. and Mecking, H. (1989). Acta metall. 37, 2017-2029. 